\title{
Le choix de la position d'un ouvrage souterrain par rapport à l'orientation des contraintes principales naturelles
}

M. AL HEIB

H. LAOUINI

J.P. PIGUET

Laboratoire de Mécanique des Terrains

École des Mines de Nancy

INERIS
Les observations récentes concernant les ruptures de roches autour des ouvrages souterrains de génie civil. minier ou pétrolier ont montré l'existence de plusieurs mécanismes de rupture. L'évaluation de la stabilité et la conception du renforcement et du soutènement de toutes les excavations passent par la connaissance des mécanismes et des modes de rupture. Dans cet article, nous développons des idées inspirées par des

observations; les observations sont confrontées à des calculs analytiques et numériques. Le tenseur final des contraintes (somme du tenseur initial et du tenseur induit) détermine le mode de rupture. Le tenseur des contraintes induites par l'excavation dépend à la fois de la géométrie de l'ouvrage (circulaire, quadrangulaire, vertical ou horizontal) et du tenseur des contraintes initiales. Une modification de l'un ou l'autre de ces deux facteurs déterminera donc en fin de compte le type de rupture. Dans les analyses de rupture en deux dimensions (problème de déformation plane, contraintes planes...), on considère que la troisième contrainte (contrainte axiale) est une contrainte intermédiaire. Nous montrerons que cette contrainte pourrait être une contrainte majeure ou mineure en fonction des deux éléments introduits cidessus. Ces analyses permettent donc de comprendre beaucoup d'observations. En retour, elles donnent des indications précieuses sur le champ des contraintes in situ, et permettent notamment de mieux interpréter les mesures des contraintes. Dans le cas où les contraintes sont connues, nous pouvons tenter de prédire le ou les types de ruptures qui pourront se produire et donc les mesures nécessaires à prendre pour les éviter.

\section{Choosing underground workings in relation to the orientation of the main naturel stresses}

Recent observations of fallures in the vicinity of underground civil engineering, mining or petroleum exploration workings have revealed the existence of several failure mechanisms. The evaluation of the stability and design of the reinforcement and support of the workings requires a knowledge of the mechanisms and modes of rupture. The observations are compared with analytical and numerical calculations. The final stresses tensor (sum of the initial tensor and induced tensor) determine the mode of rupture. The stresses tensor induced by the excavation depends both on the geometry of the workings (circular, quadrangular, vertical or horizontal) and the tensor of the initial stresses. A modification of one of these two factors will ultimately therefore determine the mode of rupture. In the analyses of two-dimensional rupture (problem of plane deformation, of plane stresses... it is considered that the third stress (axial stress) is an intermediate stress. We will show that this stress could be a major or a minor stress depending on the elements introduced above. These analysis make possible in particular to obtain the field of in situ stresses, and make possible, in particular, to obtain a better interpretation of the stress measurements. In the case where the stresses are known, we can attempt to predict the type(s) of ruptures which could occur and therefore determine the measurements which need to be taken to prevent them. 


\section{Introduction}

La stabilité des ouvrages qu'ils soient superficiels ou profonds dépend en grande partie des relations entre l'état des contraintes finales et la résistance des roches constituant le massif encaissant.

En Australie (Gale et al., 1987), dans la région sud du bassin houiller de Sydney, au cours du creusement, des cassures sont apparues au toit et au mur des voies. La position et l'importance des cassures dépendent de l'orientation de l'axe de la galerie par rapport à la plus grande contrainte horizontale. Cette instabilité est aussi rencontrée dans la majorité des bassins houillers aux USA où la contrainte horizontale naturelle est élevée.

Dans le domaine de l'industrie pétrolière (Gough et al., 1982) les puits, lors de leur forage, montrent des formes de ruptures caractéristiques telles l'ovalisation, les hors-profils ( $($ break outs $)$ ), qui fournissent une information sur le champ des contraintes en place, et l'anisotropie du champ horizontal. L'analyse de ce type de rupture a été retenue pour l'établissement de la carte mondiale des contraintes (Zobak, 1985) surtout dans des domaines où les mesures directes sont très difficiles et très chères. Plusieurs modes de rupture par cisaillement sont observés in situ. Ils dépendent de l'ordre des trois contraintes principales; (Maury et al., 1987).

De même, l'observation au laboratoire montre que les fissures microscopiques se développent et s'orientent selon l'état de chargement (contrainte principale majeure $\sigma_{1}$ et confinement $\sigma_{3}$ ).

L'état des contraintes après la réalisation d'un ouvrage dépend de plusieurs paramètres. Ces paramètres sont imposés par le type de projet, la forme de l'ouvrage et ses dimensions. L'état initial des contraintes qui dépend de la tectonique, de la topographie et des propriétés des roches est une donnée non modifiable. En revanche l'un des paramètres cités peut être modifié pour réaliser l'ouvrage dans les meilleures conditions et assurer sa stabilité. Dans certains cas où l'ouvrage risque de présenter des problèmes de stabilité, la connaissance des modes de rupture facilite la prise de décision (par exemple : choix d'un soutènement adapté). L'analyse effectuée dans cette article concerne un milieu parfaitement élastique isotrope. Mais ce travail peut être généralisé à d'autres types de comportement.

\section{2}

\section{Distribution des contraintes autour d'un ouvrage}

\section{1}

\section{L'État initial des contraintes}

Avant de réaliser un ouvrage dans un massif rocheux, les terrains sont dans un état d'équilibre. Il correspond à une distribution des contraintes dites (c naturelles» ou « initiales ». En admettant que la déformation latérale est nulle et selon la loi de Hooke

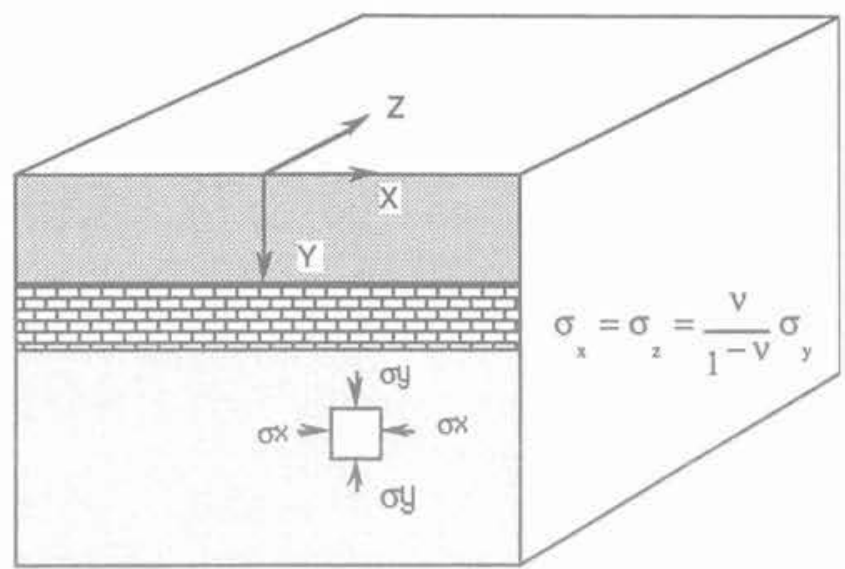

FIG. 1 État des contraintes initiales avant excavation en élasticité.

State of initial stresses before excavation in elasticity.

(Fig. 1), le champ des contraintes horizontales $\left(\sigma_{x^{\prime}} \sigma_{z}\right)$ est lié à la contrainte verticale $\sigma_{\mathrm{y}}$ et aux caractéristiques de terrains. En adoptant l'hypothèse d'une isotropie dans le plan horizontal, nous obtenons:

$$
\varepsilon_{\mathrm{x}}=\varepsilon_{\mathrm{z}}=0 \text { et } \sigma_{\mathrm{y}}=\gamma \mathrm{y}
$$

Dans ces conditions seulement les contraintes horizontales sont données par l'expression:

$$
\sigma_{x}=\sigma_{z}=K \sigma_{y}[K=v /(1-v)]
$$

(Remarque: $\operatorname{Si} \nu=0,5$ alors $\sigma_{\mathrm{x}}=\sigma_{\mathrm{y}}=\sigma_{\mathrm{z}}=\gamma \mathrm{y}$ )

$\checkmark$ coefficient de Poisson

$\gamma$ poids volumique

De récentes mesures de contraintes effectuées dans le monde ont montré, que la contrainte verticale en $\mathrm{MPa}$ (compression positive) est liée à la profondeur $\mathrm{H}$ exprimée en mètres, par la relation moyenne: $\sigma_{y}=0,027 \mathrm{H}$, ce qui vérifie globalement l'équilibre.

Le rapport $\mathrm{K}$ des contraintes verticales et horizontales dépend de nombreux facteurs: la profondeur $\mathrm{H}$, les propriétés mécaniques des terrains, I'histoire tectonique, la configuration des terrains, etc.

Une sédimentation progressive conduira à une valeur de $\mathrm{K}$ de 0,3 à 0,7 tandis qu'une tectonique horizontale très active donnera plutôt des valeurs de $\mathrm{K}$ comprise entre 0,7 et 2 . La contrainte horizontale moyenne varie de 0,5 à 3,0 fois la contrainte verticale pour une même profondeur (Fig. 2). Les deux contraintes principales horizontales peuvent être totalement différentes.

\section{2}

\section{État final des contraintes}

Le creusement d'un ouvrage dans un massif rocheux crée un nouvel état des contraintes. Il en résulte des contraintes nouvelles de traction, de cisaillement, et de compression. Sous leur action, les roches au voisinage de l'excavation se déforment, se déplacent et se fissurent. Il est intéressant de connaître le ou les types de ruptures, ainsi que les plans dans lesquels les fractures se propagent. Pour cela, il faudra connaitre les orientations des contraintes principales. Le tenseur initial, l'orientation de l'axe de l'ouvrage et les caractéristiques du massif sont les éléments nécessaires pour déterminer le tenseur final des contraintes. 
$K$ : stresses ratio $\left.\phi_{v} / \sigma_{h}\right)$

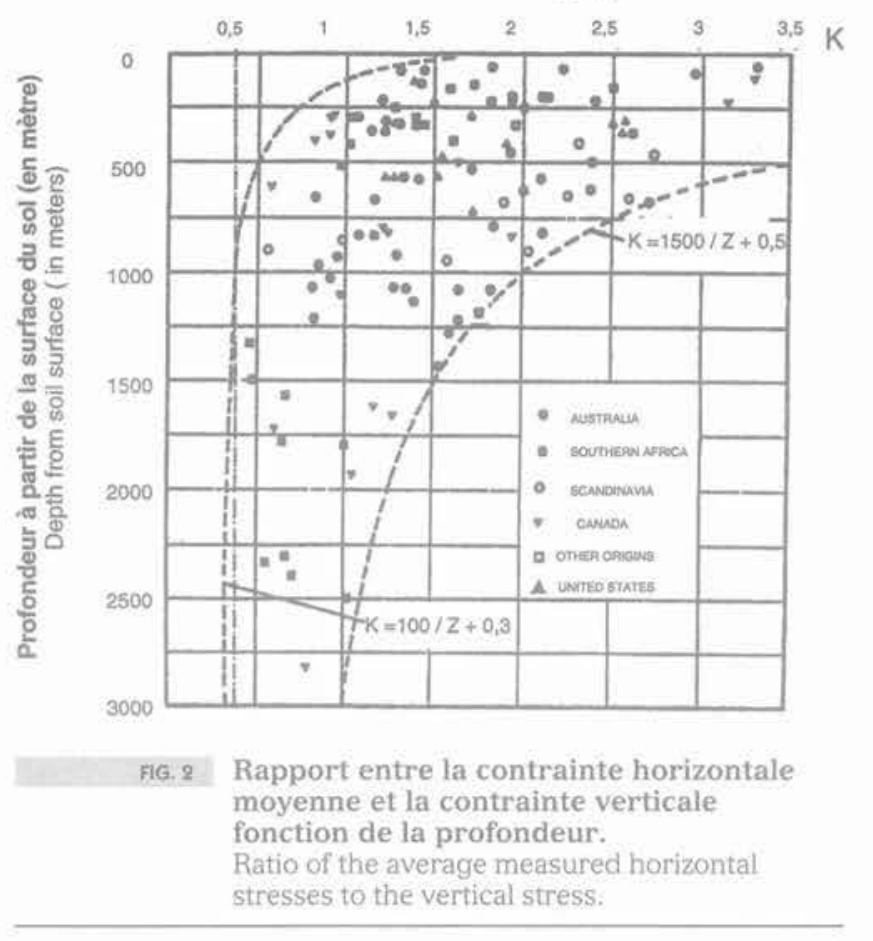

Orientation de l'ouvrage par rapport aux contraintes initiales

Dans le tenseur initial principal des contraintes, on distingue trois directions: celles des contraintes principales majeure, intermédiaire et mineure. Les contraintes induites dépendent aussi de l'orientation de l'axe de l'ouvrage par rapport aux directions des contraintes principales.

Dans le cas général où le tenseur des contraintes initiales est anisotrope, les directions de ces trois contraintes principales sont présentées sur la figure 3 . Leurs amplitudes relatives, sont 1, 2 et 3 respectivement. Les galeries, supposées cylindriques, sont décrites dans un repère local où oz désigne toujours la direction longitudinale, ox et oy les directions transversales parallèles aux directions principales initiales. Nous allons étudier successivement les cas oủ l'axe de la galerie coïncide avec la direction de l'une des contraintes principales.

Pour l'analyse des contraintes finales, dans chaque cas, nous nous placerons dans un repère de coordonnées cylindriques $(\mathrm{z}$, direction longitudinale, $r$ et $\theta$ directions radiale et orthoradiale).

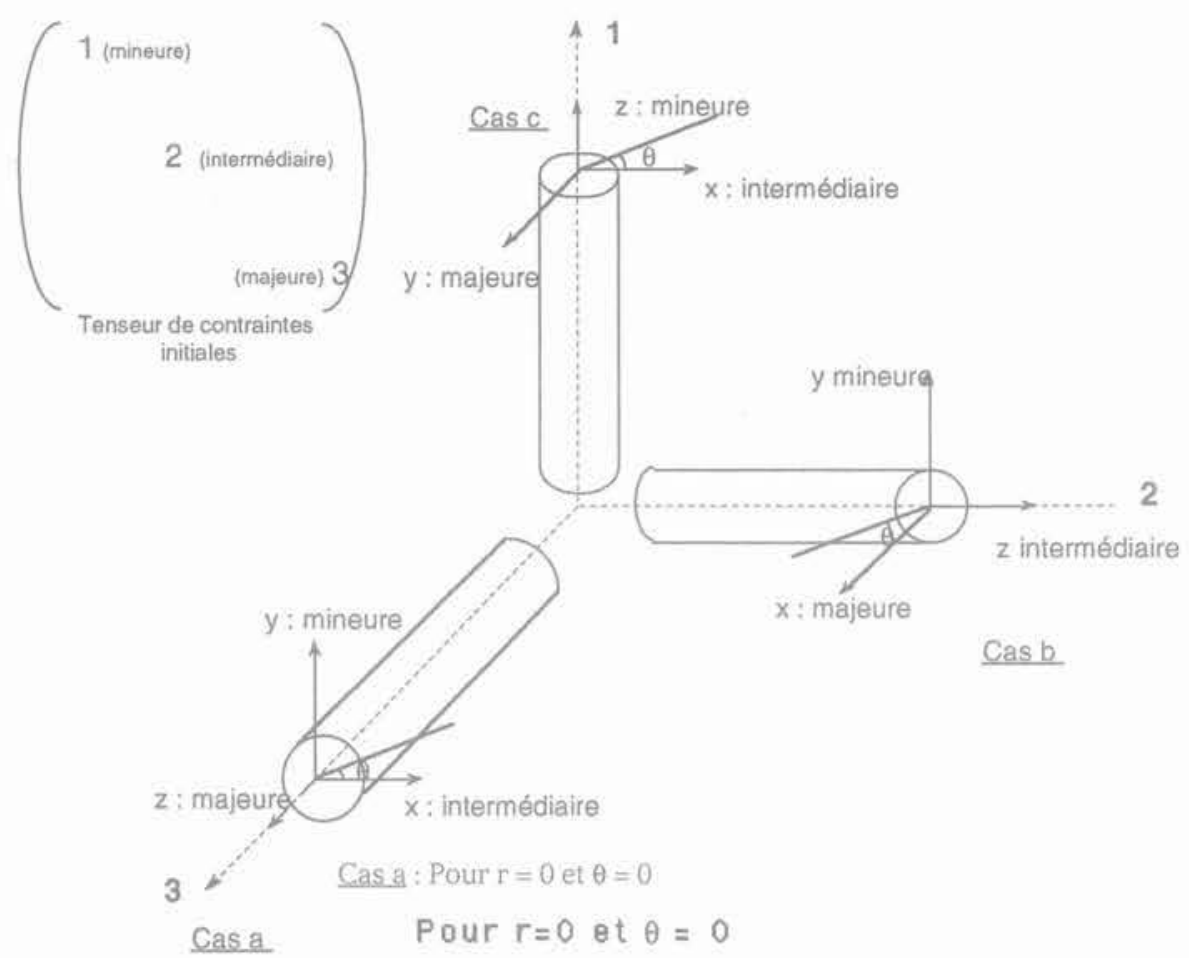

a - la galerie dans l'axe de la contrainte majeure:

Contraintes initiales : $\sigma \mathrm{y}=1, \sigma \mathrm{x}=2$ et $\sigma \mathrm{z}=3$

Contraintes finales : $\sigma \theta=1$ et $\sigma z=$ contrainte majeure $=2,5$

a-the opening in major stress axis :

Initial stresses : $\sigma y=1, \sigma x=2$ and $\sigma z=3$

Final stresses : $\sigma \theta=1$ and $\sigma z=$ major stress $=2,5$

$\mathrm{b}$ - la galerie dans l'axe de la contrainte intermédiaire:

Contraintes initiales : $\sigma \mathrm{y}=1, \sigma \mathrm{x}=3$ et $\sigma \mathrm{z}=2$

Contraintes finales : $\sigma \theta=0$ et $\sigma \mathrm{z}=$ contrainte majeure $=1$

$c$ - la galerie dans l'axe de la contrainte mineure:

Contraintes initiales : $\sigma \mathrm{y}=3, \sigma \mathrm{x}=2$ et $\sigma z=1$

$\mathrm{b}$ - the opening in intermediate stress axis:

Initial stresses : $\sigma \mathrm{y}=1, \sigma \mathrm{x}=3$ and $\sigma z=2$

Final stresses : $\sigma \theta=0$ and $\sigma z=$ major stress $=1$

$c$ - the opening in minor stress axis :

Initial stresses: $\sigma \mathrm{y}=3, \sigma \mathrm{x}=2$ and $\sigma \mathrm{z}=1$

Final stresses : $\sigma z=1,5$ and $\sigma \theta=$ major stress $=7,7$

FIG.3 Contraintes finales en fonction de l'orientation de l'ouvrage par rapport au tenseur des contraintes principales.

Final stresses at boundary of opening as a function of the orientation of the principal stresses tensor. 
Cas a: L'axe de la galerie correspond à la direction de la contrainte majeure:

Dans ce cas la contrainte initiale selon $x$ est égale à 2 , selon y est égale à 1 et selon $z$ est égale à 3 . Les contraintes finales pour $\mathrm{r}=\mathrm{a}$ et $\theta=0$ sont $\sigma_{\mathrm{r}}=0$ et $\sigma_{\mathrm{\theta}}=$ 1 et $\sigma_{2}=2,5$. Donc la direction de la contrainte principale majeure après l'excavation correspond à la direction axiale.

Cas b: L'axe de la galerie correspond à la direction de la contrainte intermédiaire :

Dans ce cas, la contrainte initiale selon $x$ est égale à 3, la contrainte selon y est égale à 1 et la contrainte selon $z$ est égale à 2 . Les contraintes finales pour les mêmes conditions sont $\sigma_{\mathrm{r}}=0$ et $\sigma_{\mathrm{\theta}}=0$ et $\sigma_{\mathrm{z}}=1$. Nous constatons que la valeur de la contrainte principale majeure est égale à 1 et sa direction correspond à la direction axiale.

Cas c: L'axe de la galerie correspond à la direction de la contrainte mineure.

Nous pouvons constater que les contraintes initiales sont égales à 2 selon $x, 3$ selon y et 1 selon l'axe $z$ de la galerie. Les valeurs des contraintes finales pour le même point sont les suivantes: $\sigma_{r}=0, \sigma_{\theta}=7$ et la contrainte axiale $\sigma_{\gamma}=1,5$. La contrainte principale majeure est égale à 7 et sa direction correspond à l'axe oy.

Pour ces cas particuliers, nous constatons que la valeur de la contrainte principale majeure totale (induite + initiale) est fonction de l'orientation de la galerie, et varie entre 1 et 7 . De plus, la direction de cette contrainte n'est pas toujours la même.

Si on suppose un critère de rupture comme celui présenté sur la figure 4, nous constatons qu'au point de la paroi considéré, le critère est dépassé dans le troisième cas, juste atteint dans le premier cas et non obtenu dans le second cas. Donc le bon choix de l'orientation de l'ouvrage par rapport aux directions de contraintes permet d'éviter la rupture en un point de la paroi. Dans le cas où la direction de l'ouvrage est imposée, un soutènement efficace et qui s'adapte avec les modes de ruptures possibles, est nécessaire.

\section{4}

\section{Forme de la galerie à réaliser}

Dans le cas d'un ouvrage circulaire horizontal, sans soutènement, creusé dans un massif soumis au préalable à un état de contrainte caractérisé par ses contraintes principales initiales, les contraintes après

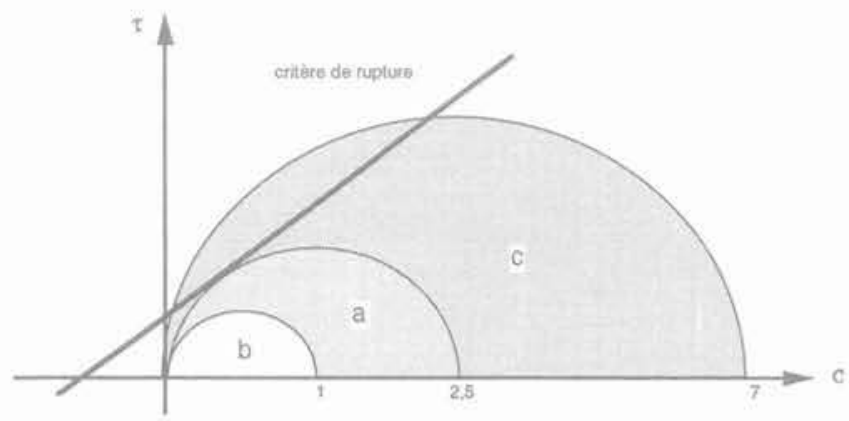

FIG. 4 Cercles de Mohr pour les trois cas par rapport à un critère de rupture. Mohr circles for three cases plotted with a criterion failure. creusement peuvent être données par les expressions de Kirsch. Dans le cas d'un ouvrage non circulaire, en déformation plane, on ajoute des coefficients de forme à ces expressions.

Ces coefficients A et B sont liés à la forme particulière de la galerie; ils varient entre 1,5 et 5 et ont été déterminés par Hoek \& Brown, 1980 (Fig. 5).

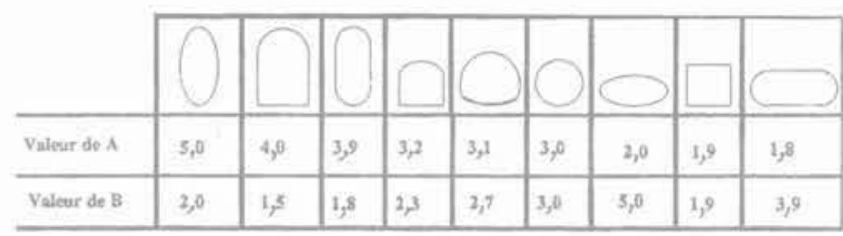

FG.5 Valeur des coefficients de forme (d'après Hoek \& Brown, 1980).

Ratio of applied stresses to shape excavations.

Sur la base d'un calcul élastique, en coordonnées cylindriques $(r, \theta)$ en se limitant à deux points pour cette analyse, la contrainte orthoradiale $\sigma_{8}$ (contrainte horizontale en clé et verticale en piédroit, lors du creusement est, pour $r=0$ :

en piédroit $(\theta=0)$ :

$$
\begin{aligned}
& \sigma_{\theta}=(B-K) \sigma_{v} \\
& \sigma_{z}=[K(\alpha-2 v)+v(B-1)] \sigma_{y}
\end{aligned}
$$

en voûte $(\theta=\pi / 2)$;

$$
\begin{aligned}
& \sigma_{\theta}=(A K-1) \sigma_{v} \\
& \sigma_{2}=[K((A-1) v+\alpha)-2 v] \sigma_{v}
\end{aligned}
$$

avec $\alpha=K^{\prime} / K$

$(\mathrm{K}=$ contrainte transversale horizontale initiale/ contrainte verticale initiale)

( $\mathrm{K}^{\prime}=$ contrainte horizontale longitudinale initiale/ contrainte verticale initiale)

On remarque que les contraintes axiale $\left(\sigma_{z}\right)$ et orthoradiale $\left(\sigma_{\theta}\right)$ sont fonction d'une part, des coefficients $A$ et $\mathrm{B}$ liés à la forme de la section de l'ouvrage, et d'autre part, du coefficient $\alpha$ mettant en évidence l'état d'anisotropie dans le plan horizontal. La contrainte axiale longitudinale $\left(\sigma_{z}\right)$ dépend aussi du coefficient de Poisson $v$,

Pour étudier cet aspect nous avons représenté graphiquement $\alpha$ (Fig, 6) en fonction de A, B en paroi et en voute, pour différentes valeurs de $\mathrm{K}$. Cela permet de définir les domaines pour lesquels la contrainte axiale (longitudinale) serait majeure pour des valeurs supérieures à $\alpha$ que ce soit en voûte ou en paroi.

Nous constatons que:

En voûte, la contrainte axiale est majeure (par conséquent déterminante pour l'apparition de zones où le critère de rupture est dépassé):

- pour les galeries de forme élancée verticalement (A fort), pour lesquelles les contraintes horizontales initiales sont plutôt anisotropes $(\alpha>1)$ et plutôt dominantes par rapport à la contrainte verticale;

- pour les galeries de forme aplatie (B fort et A faible), pour lesquelles les contraintes initiales horizontales sont plutôt isotropes.

En paroi, la contrainte axiale est majeure:

- pour des faibles valeurs de A et B et une faible anisotropie des contraintes horizontales $(\alpha=1)$;

- pour un état des contraintes horizontales initial fortement anisotrope et faible par rapport à la contrainte verticale initiale ( $\mathrm{K}$ faible); 

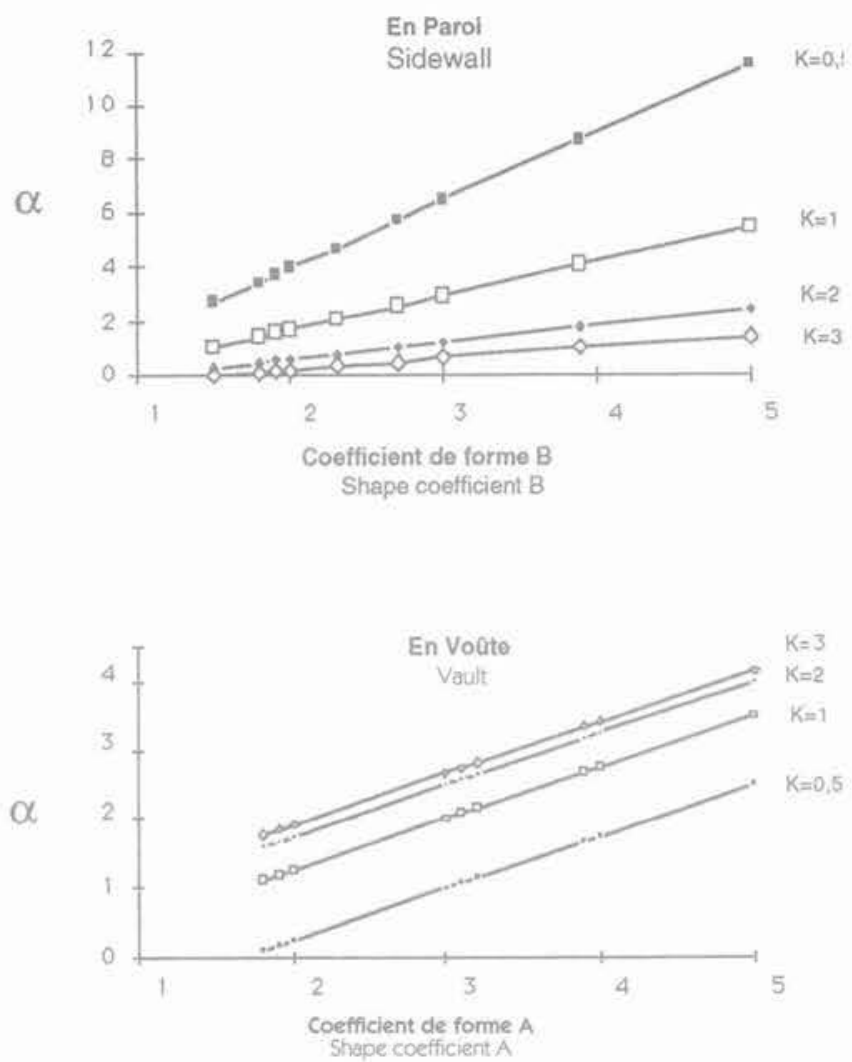

FG.6 Rapport des contraintes horizontales initiales $\alpha$ en fonction de la forme de T'ouvrage pour lesquels la contrainte longitudinale est majeure.

Horizontal stresses ratio $\alpha$ function of shape excavation for which longitudinal stress is major.

- pour les galeries de forme élancée verticalement et pour lesquelles l'état de contrainte horizontale est anisotrope.

L'influence du coefficient de Poisson est moins importante. Elle concerne surtout des contraintes horizontales relativement faibles soit en voûte soit en paroi.

Connaissant A, B, K et $\alpha$ on peut déterminer la contrainte majeure. Les contraintes mineure et majeure doivent être prises en compte dans les calculs de stabilité.

\section{5}

\section{Dimensions de l'ouvrage}

Dans le cas d'un ouvrage circulaire, en milieu élastique isotrope, les dimensions de l'ouvrage n'interviennent pas, en revanche pour un ouvrage rectangulaire le rapport d'élancement intervient pour déterminer les contraintes finales.

Nous allons déterminer le rapport des contraintes permettant à la contrainte axiale d'être une contrainte majeure ou mineure. Nous avons adopté une méthode numérique (éléments finis) pour analyser les contraintes autour d'une galerie rectangulaire creusée dans un milieu élastique isotrope. Nous supposons que la galerie, de largeur " $L$ ) et de hauteur ( $h$ ) est creusée à $\mathrm{H}$ mètres de profondeur. Les contraintes initiales sont, comme dans les paragraphes précédents : une contrainte verticale $\sigma y$ (poids propre) et des contraintes horizontales (transversale ox et longitudinale $\sigma z$ ) pro- portionnelles à la contrainte verticale : $\sigma_{\mathrm{x}}=\mathrm{K} \sigma_{\mathrm{y}}$ et $\sigma_{z}=K \sigma_{y}$

Nous avons distingué l'état des contraintes en paroi et au toit. Les paramètres qui interviennent dans ce cas sont le rapport largeur/hauteur $(\mathrm{L} / \mathrm{h})$ et le rapport des contraintes $((\mathrm{K})$,

Nous avons d'abord fait varier le rapport $(L / h)$ entre 0,5 et 3 . Pour chaque valeur du rapport, nous avons cherché le rapport des contraintes « $\mathrm{K}$ ) dit « limite» correspondant à une contrainte axiale selon $\mathrm{z}$ majeure ou mineure.

Nous constatons qu'en paroi la valeur de ( K limite » augmente au fur et à mesure que la largeur de la galerie est importante (Fig. 7). Autrement dit, la contrainte axiale est une contrainte majeure pour des valeurs de $\mathrm{K}$ supérieures à « $\mathrm{K}$ limite $»$. En ce point de la galerie, les trois composantes des contraintes sont en compression, le risque de rupture est dû au cisaillement.

$$
\sigma_{z}>\sigma_{y}>\sigma_{x}
$$

Pour les ouvrages de grande portée (largeur supérieure ou égale à deux fois la hauteur), nous obtenons trois valeurs différentes de " $\mathrm{K}$ limite», deux en voûte selon que les contraintes horizontales sont faibles ou importantes, et l'autre en paroi.

Nous allons analyser le cas d'une galerie pour laquelle le rapport $(\mathrm{L} / \mathrm{h})$ est égal à 3 et elle est à $1000 \mathrm{~m}$ de profondeur (Fig. 8):

\section{Au toit}

Cas a: nous constatons que pour des valeurs de $((\mathrm{K}))$ inférieures à 0,7 , la contrainte transversale horizontale
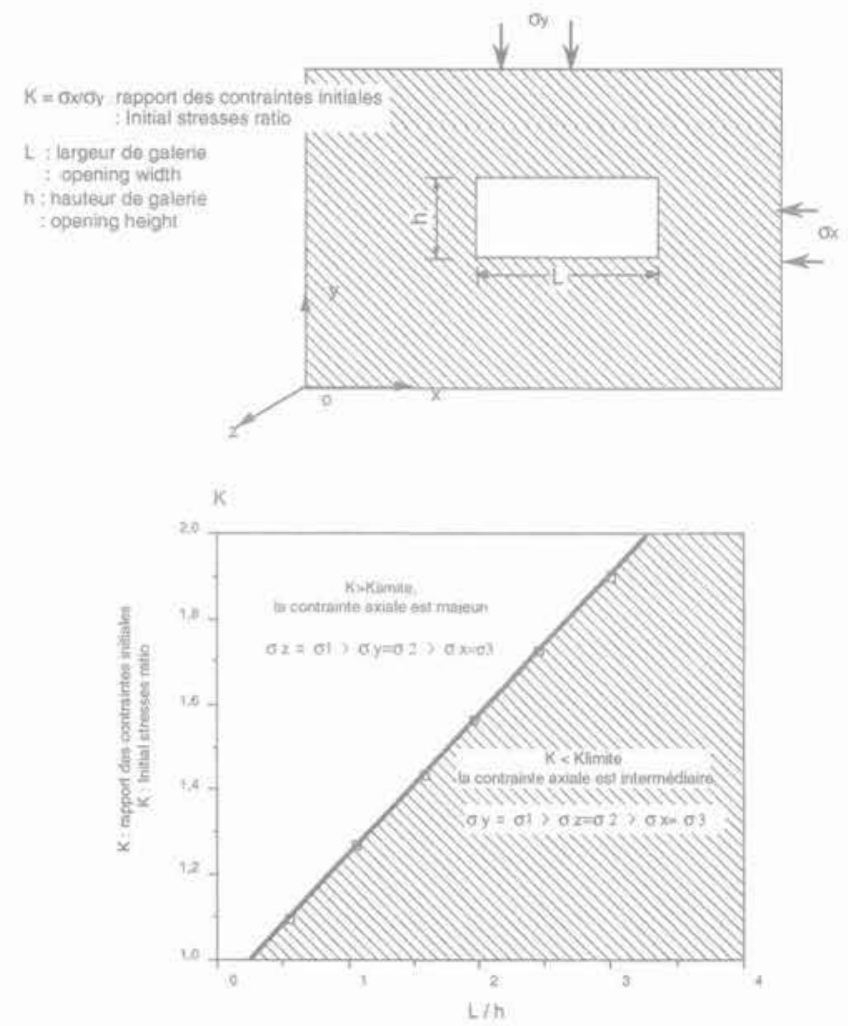

FG. 7 Valeur de $\mathrm{K}$ limite en fonction des dimensions de la galerie pour laquelle la contrainte axiale finale est majeure à la paroi.

Limit value of $\mathrm{K}$ depending on dimensions opening for which final axial stress is major in sidewall. 

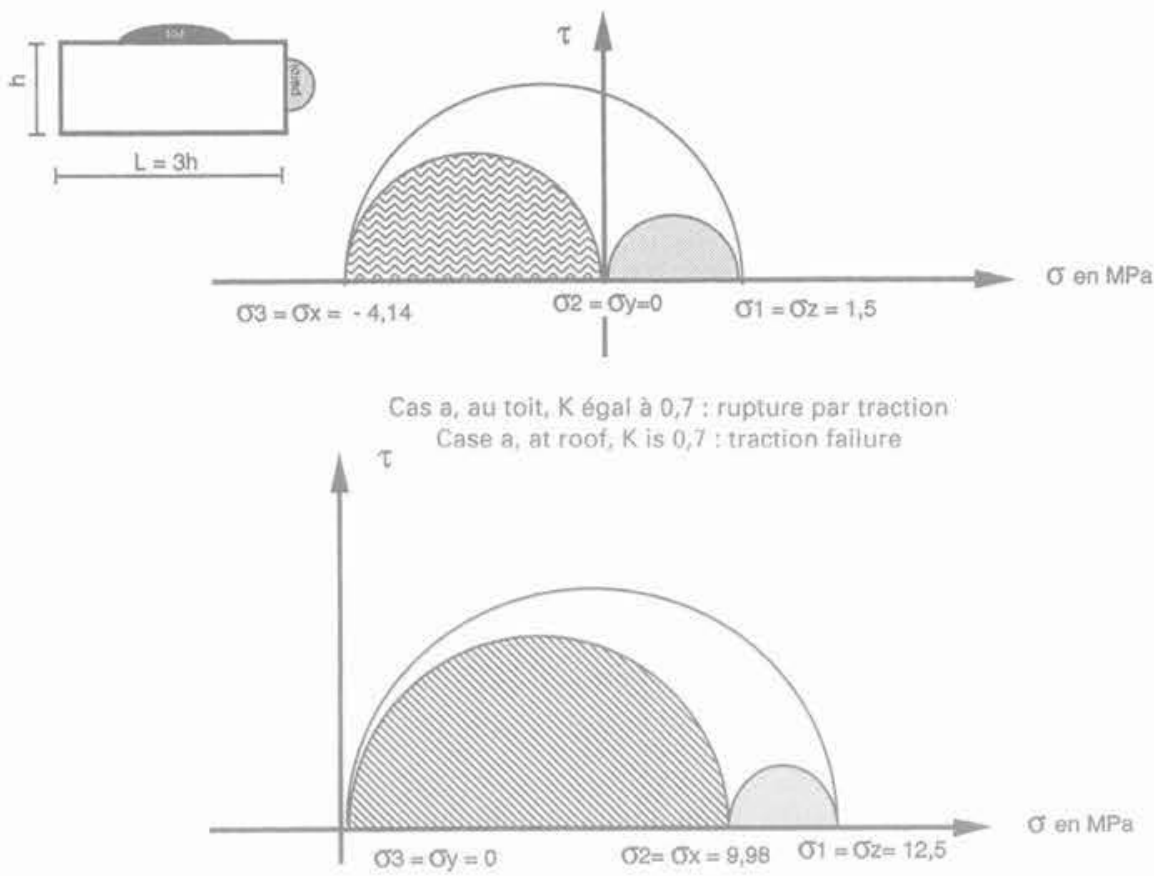

Cas b, au toit, $K$ égal à 1,25: rupture par cisaillement Case, at roof, $K$ is 1,25 ; shear failure

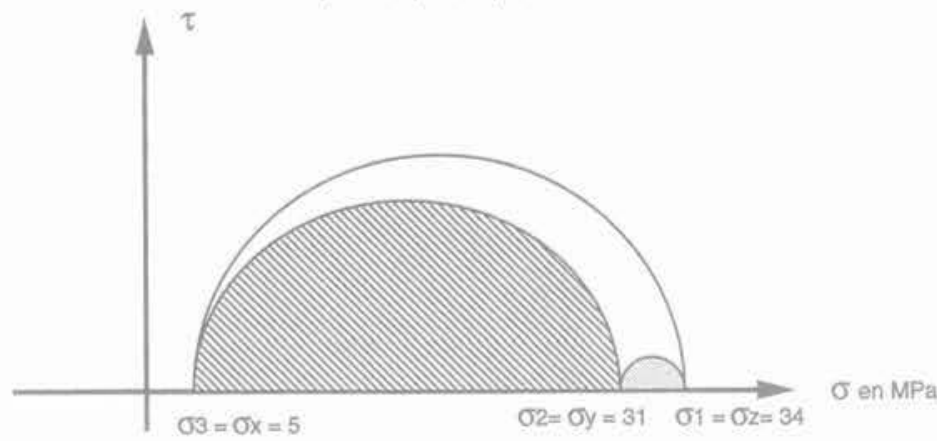

Cas C, aux parements, $K$ égal à 2 : nupture par cisaillement Case c, at sidewail, Kis 2: shear failure

FG. 8 Types de rupture d'une galerie rectangulaire en fonction de rapport des contraintes verticale et horizontale.

Failure modes for rectangular opening fonction of vertical and horizontal stresses ratio.

$\sigma x$ est en traction, elle est la contrainte principale mineure $\left(\sigma_{3}\right)$. La contrainte transversale verticale $\sigma y$ est intermédiaire $\left(\sigma_{2}\right)$. La contrainte axiale longitudinale $\sigma z$ est une contrainte principale majeure de compression. La rupture est une rupture par extension selon x.

Cas b: il correspond à une contrainte horizontale initiale forte, la contrainte horizontale transversale n'est plus en traction au toit de la galerie. Elle est une contrainte de compression intermédiaire $\left(\sigma_{2}\right)$. Les deux contraintes principales sont la contrainte verticale mineure $\left(\sigma_{3}\right)$ et la contrainte axiale majeure $\left(\sigma_{1}\right)$. Le grand cercle de Mohr est composé d'une contrainte verticale quasi nulle et d'une contrainte axiale de compression (analogie avec l'essai de compression simple).

\section{En paroi}

Cas c: la valeur de « $\mathrm{K}$ limite » est égale à 2. La contrainte horizontale transversale $\sigma x$ est la contrainte mineure de compression. La contrainte verticale $\sigma y$ est la contrainte intermédiaire, et la contrainte longitudinale oz est la contrainte principale majeure.

Pour les ouvrages rectangulaires la contrainte axiale pourra donc être une contrainte majeure ou mineure dans les zones les plus exposées à la rupture. Le rapport des contraintes initiales verticales sur horizontales "K limite» détermine la situation dans laquelle cette contrainte est extrême. Plusieurs modes de rupture sont possibles.

\section{Conclusion}

L'évaluation de la stabilité et la conception du renforcement et du soutènement de toutes les excavations passent par la connaissance des mécanismes et des modes de rupture. Des résultats importants semblent 
déjà acquis dans certains domaines pour caractériser et identifier clairement les modes de rupture. Nous avons montré que plusieurs types de ruptures peuvent apparaitre autour d'une excavation, qu'elle soit circulaire (galerie, puits) ou rectangulaire. Nous avons constaté que ces modes de rupture dépendent de l'orientation des contraintes principales par rapport à l'ouvrage et de leur valeur. Dans les problèmes de déformation plane, la contrainte axiale n'est pas toujours une contrainte intermédiaire. Il est tout à fait possible qu'elle soit une contrainte majeure ou mineure surtout pour des contraintes horizontales initiales élevées. Dans ce cas, les modes de rupture dépendent de la contrainte axiale. Ces analyses permettent de com- prendre beaucoup d'observations. En retour, elles donnent des indications précieuses sur le champ des contraintes in situ. Inversement, quand les contraintes sont connues, nous pouvons proposer le ou les types de ruptures qui pourront se produire et donc les mesures nécessaires pour les éviter. La mine avec ses galeries et ses ouvrages très variés présente des cas qui permettent de faire progresser la connaissance sur les modes de ruptures. Nous avons constaté que pour chaque type d'ouvrages, deux ou trois modes de rupture sont possibles. Par conséquent, nous pouvons mieux comprendre les phénomènes observés et adapter les modes de soutènements capables d'assurer la sécurité des ouvrages.

\section{Bibliographie}

Gale W.J. et Blackwood RI (1987) - Stress distribution and rock failure around coal mine roadways. Int. J. Rock Mech. Min. Sci. \& Geomech. Abstr. Vol. 24, $\mathrm{n}^{\circ} 3$, pp. 165-173.

Guenot A. (1989) - Borehole Breakouts and Stress Fields. Int. J. Rock Mech. Min. Sci \& Geomech, Abstr. Vol. 26, no3 $3 / 4$, pp. 185-195.

Jaeger J.C., Cook N.G.W. (1979) - Fundamentals of Rock mechanics. Methuen and $\mathrm{co}$, London.

Guenot A. (1987) - Contrainte et rupture des forages pétroliers. 6th Int. conf. Rock Mech. Vol. 1, pp. 109-118. Ed. Balkema, Rotterdam.

Santareli F.J., Brown E.T. (1989) - Failure of three sedimentary rocks in triaxial and hollow cylinder compression test. int. J. Rock Mech. Min. Sci \& Geomech. Abstr. Vol. 26, n 5, pp. 401-413.

Maury V.M. Sauzy J.-M. (1989) - Rupture de puits provoquée par glissement sur faille : cas vécu, mécanisme, remèdes, conséquences. Rock at Great Depth, Balkema, Rotterdam, pp. 871-876. Asszonyi Cs. Richter R. (1979) - The continuum theory of rock mechanics. Serie on rock and soil Mechanic. Vol. 4s-, Trans Tech Publications, Germany.

Piguet J.-P. (1983) - La modélisation en mécanique des terrains et son application à l'exploitation Minière. Thèse de Dr. ès Science. Laboratoire de Mécanique des Terrains. INPL. Nancy.

Brady B.H.G.. Brown E.T. (1985) - Rock Mechanics for underground Mining. George Allen \& Unwin Ed. London.
Zoback M.D. (1985) - Wellbore breakouts and in situ stress. J. of Geoph. Res, 90 $\mathrm{n}^{\circ} \mathrm{B} 75523-5530$

Gough D.I. \& Bell J.S. (1982)-Stress orientation from borehole wall fracture with examples from Colorado, east Texas, and northern Canada. Can J. Earth Sci. 19. pp. 1358-1370.

Laouni H. (1993) - Developpement d'un système expert d'aide à la définition d'un programme de reconnaissance pour les tunnels. Doct. INPL (École des Mines de Nancy).

Al Heib M.M. (1993) - Les nouvelles méthodes de modélisation numériques et le volume d'influence des exploitations minières en conditions complexes. Doct. INPL (Ecole des Mines de Nancy). 